\title{
IR based Haemoglobin Measurement using Non Invasive Technique and its Classification
}

\author{
Prashant Chougule, Pooja Shirodkar, Gauresh Gopal Shetgaonkar Sitaram Alias, \\ Niharika Samant, Chaitali Haldankar, \\ Electronics and Communications Engineering Department, \\ Agnel Institute of Technology and Design, Goa
}

\begin{abstract}
Hemoglobin is the protein molecule in red blood cells that carries oxygen from the lungs to the body's tissues and returns carbon dioxide from the tissues back to the lungs.

Haemoglobin consist of four protein molecules (globulin chains) that are linked together. The normal adult haemoglobin (Hbg) molecule contains two alpha-globulin chains and two betaglobulin chains. Invasive method comprises of physical examination and credentialises the colour of a drop of blood on filter paper to a standardized colour comparison chart[3].

Here is an attempt to design a system for non-invasive haemoglobin monitoring and classification . A non invasive haemoglobin monitoring using infrared is showcased. It includes near infrared radiation on a index finger of your hand and sensing the resultant radiation after absorbance by haemoglobin . The haemoglobin count is measured across to the voltage level through a chain of observations and results are obtained .The results are further used for analysing the various haemoglobin related diseases prevalent in women[3].
\end{abstract}

Keywords: Non invasive, monitoring, fuzzy logic, classification

\section{INTRODUCTION}

According to World Health Organisation the normal range for $\mathrm{Hb}$ Is For men : $13.5 \mathrm{~g} / \mathrm{dl}$ to $17.5 \mathrm{~g} / \mathrm{dl}$ and For women : $12.0 \mathrm{~g} / \mathrm{dl}$ to $15.5 \mathrm{~g} / \mathrm{dl}$

$\mathrm{Hb}$ values can be lower or higher than the normal range resulting in different types of diseases. If $\mathrm{Hb}$ concentration is lower than normal levels then it is termed as anaemia whereas high hb levels is termed as polycythaemia.

\section{MANUSCRIPTS}

\section{A. Hemoglobin}

Hemoglobin is present in the red blood cells of the body. Absorption of light by oxygenated and deoxygenated hemoglobim is measured by wavelengths $940 \mathrm{~nm}$ and $660 \mathrm{~nm}$ respectively.

Each red blood cell (RBC) contains approximately 280 million hemoglobin molecules.

The main task of hemoglobin is to carry oxygen from the lungs to the tissues and then transport $\mathrm{CO} 2$ from the different tissues to the lungs. One hemoglobin molecule has the ability to carry up to 4 oxygen molecules. There are two main components of hemoglobin: oxyhemoglobin, which is saturated with oxygen molecules and deoxyhemoglobin (also is called reduced hemoglobin), which is desaturated with oxygen molecules. Oxyhemoglobin is highly associated for oxygen than deoxyhemoglobin, and deoxyhemoglobin is highly associated to $\mathrm{CO} 2$ than oxyhemoglobin. Therefore, oxygen combines to oxyhemoglobin in the lungs and is then transferred through the blood stream until it reaches various tissues. There, the oxygen is released to myoglobin, which then carries it to the mitochondria, and thus used for aerobic respiration. In exchange, deoxyhemoglobin picks up 2 protons and 2 molecules of $\mathrm{CO} 2$ and returns to the lungs, where the $\mathrm{CO} 2$ is set out through exhalation.

\section{B. Advantages of Non-Invasive Techniques}

- Needle free, painless testing system.

- Eliminates possible infections.

- Advantageous to the hemophobic and diabetic patients.

- No need of expertise

- Device is portable

- Cost effective

- Real time monitoring system

\section{Description of Block diagram}

The block diagram is shown below for the detection

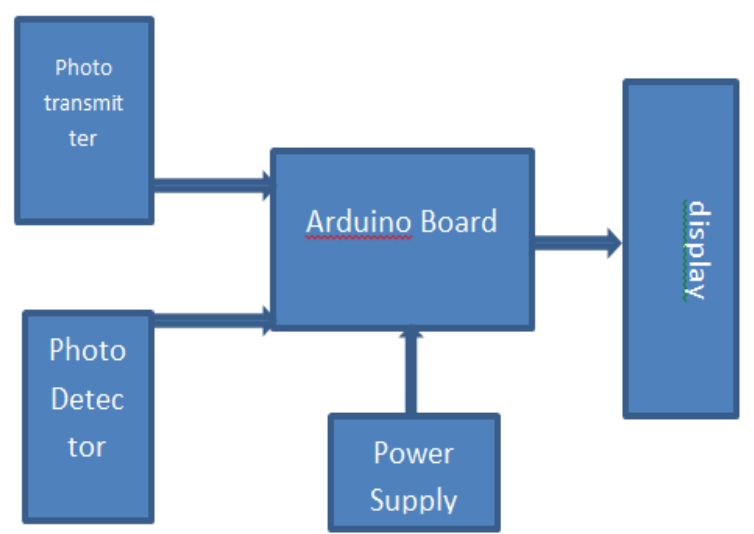

D. Hardware analysis

Figure 1: Block diagram

Table 1: Obtained Avg voltage levels for $\mathrm{Hb}$ count

\begin{tabular}{|l|l|l|l|l|l|l|l|}
\hline $\begin{array}{l}\text { Sr. } \\
\mathrm{N} \\
\mathrm{o}\end{array}$ & $\begin{array}{l}\text { Femal } \\
\text { e Age } \\
\text { Group }\end{array}$ & $\begin{array}{l}\mathrm{Hb} \\
\mathrm{Cou} \\
\mathrm{nt}(\mathrm{m} \\
\mathrm{g} / \mathrm{dl})\end{array}$ & $\begin{array}{l}\mathrm{R} 1 \mathrm{in} \\
\mathrm{mV}\end{array}$ & $\begin{array}{l}\mathrm{R} 2 \\
\mathrm{in} \\
\mathrm{mV}\end{array}$ & $\begin{array}{l}\mathrm{R} 3 \mathrm{in} \\
\mathrm{mV}\end{array}$ & $\begin{array}{l}\text { Readin } \\
\mathrm{g} \mathrm{4} \mathrm{in} \\
\mathrm{mV}\end{array}$ & $\begin{array}{l}\text { Avg } \\
\text { output } \\
\text { voltage } \\
\text { in mV }\end{array}$ \\
\hline 1 & $\begin{array}{l}10- \\
12 \mathrm{yrs}\end{array}$ & 16.6 & 5.0 & 5.2 & 5.3 & 5.0 & \\
\hline 2 & $\begin{array}{l}10- \\
12 \mathrm{yrs}\end{array}$ & 15.3 & 5.0 & 5.1 & 5.1 & 5.0 & 5.05 \\
\hline 3 & $\begin{array}{l}12- \\
15 \mathrm{yrs}\end{array}$ & 16.7 & 4.7 & 4.5 & 4.6 & 4.6 & 4.6 \\
\hline 4 & $\begin{array}{l}12- \\
15 \mathrm{yrs}\end{array}$ & 15.6 & 5.0 & 5.1 & 5.1 & 5.1 & 5.07 \\
\hline
\end{tabular}




\begin{tabular}{|c|c|c|c|c|c|c|c|}
\hline 5 & $\begin{array}{l}12- \\
15 \mathrm{yrs}\end{array}$ & 14.3 & 6.2 & 6.3 & 6.3 & 6.3 & 6.3 \\
\hline 6 & $\begin{array}{l}12- \\
15 \mathrm{yrs}\end{array}$ & 14.8 & 6.0 & 6.0 & 6.1 & 6.0 & 6.0 \\
\hline 7 & $\begin{array}{l}12- \\
15 \mathrm{yrs}\end{array}$ & 16 & 4.3 & 4.4 & 4.4 & 4.3 & 4.3 \\
\hline 8 & $\begin{array}{l}15- \\
17 \mathrm{yrs}\end{array}$ & $\begin{array}{l}16.3 \\
3 \\
\end{array}$ & 4.3 & 4.2 & 4.2 & 4.2 & 4.2 \\
\hline 9 & $\begin{array}{l}15- \\
17 \mathrm{yrs}\end{array}$ & $\begin{array}{l}15.3 \\
3 \\
\end{array}$ & 5.6 & 5.7 & 5.7 & 5.6 & 5.6 \\
\hline 10 & $\begin{array}{l}15- \\
17 \mathrm{yrs}\end{array}$ & 17.2 & 4.9 & 4.8 & 4.8 & 5.0 & 4.87 \\
\hline 11 & $\begin{array}{l}15- \\
17 \mathrm{yrs}\end{array}$ & 16.5 & 4.1 & 4.1 & 4.1 & 4.3 & 4.1 \\
\hline 12 & $\begin{array}{l}15- \\
17 \mathrm{yrs}\end{array}$ & $\begin{array}{l}13.2 \\
2 \\
\end{array}$ & 6.4 & 6.3 & 6.4 & 6.4 & 6.4 \\
\hline 13 & $\begin{array}{l}15- \\
17 \mathrm{yrs}\end{array}$ & $\begin{array}{l}13.6 \\
7\end{array}$ & 6.2 & 6.3 & 6.3 & 6.2 & 6.2 \\
\hline 14 & $\begin{array}{l}15- \\
17 \mathrm{yrs}\end{array}$ & 14.4 & 6.2 & 6.1 & 6.2 & 6.2 & 6.2 \\
\hline 15 & $\begin{array}{l}15- \\
17 \text { yrs }\end{array}$ & 15.2 & 5.5 & 5.4 & 5.5 & 5.5 & 5.5 \\
\hline 16 & $\begin{array}{l}15- \\
17 \mathrm{yrs}\end{array}$ & 15.5 & 5.0 & 4.9 & 4.9 & 4.8 & 4.9 \\
\hline 17 & $\begin{array}{l}15- \\
17 \mathrm{yrs}\end{array}$ & 16.1 & 4.7 & 4.6 & 4.5 & 4.7 & 4.6 \\
\hline 18 & $\begin{array}{l}15- \\
17 \mathrm{yrs}\end{array}$ & $\begin{array}{l}16.0 \\
2\end{array}$ & 4.5 & 4.7 & 4.7 & 4.5 & 4.6 \\
\hline 19 & $\begin{array}{l}15- \\
17 \mathrm{yrs}\end{array}$ & 15.3 & 5.5 & 5.5 & 5.6 & 5.5 & 5.5 \\
\hline 20 & $\begin{array}{l}15- \\
17 \mathrm{yrs}\end{array}$ & 16.2 & 4.8 & 4.5 & 4.8 & 4.8 & 4.8 \\
\hline 21 & $\begin{array}{l}18- \\
25 \mathrm{yrs}\end{array}$ & 11 & 7.2 & 7.1 & 7.1 & 7.1 & 7.1 \\
\hline 22 & $\begin{array}{l}18- \\
25 \mathrm{yrs}\end{array}$ & 11.6 & 7.1 & 7.2 & 7.2 & 7.3 & 7.2 \\
\hline 23 & $\begin{array}{l}18- \\
25 \mathrm{yrs}\end{array}$ & 12.3 & 6.7 & 6.8 & 6.6 & 6.7 & 6.7 \\
\hline 24 & $\begin{array}{l}18- \\
25 \mathrm{yrs}\end{array}$ & 12.7 & 6.7 & 6.7 & 6.5 & 6.6 & 6.7 \\
\hline 25 & $\begin{array}{l}18- \\
25 \mathrm{yrs}\end{array}$ & 12.8 & 6.6 & 6.4 & 6.4 & 6.1 & 6.3 \\
\hline 26 & $\begin{array}{l}18- \\
25 \mathrm{yrs}\end{array}$ & 13.2 & 6.4 & 6.3 & 6.4 & 6.4 & 6.4 \\
\hline 27 & $\begin{array}{l}18- \\
25 \mathrm{yrs}\end{array}$ & 13.6 & 6.5 & 6.4 & 6.3 & 6.3 & 6.4 \\
\hline 28 & $\begin{array}{l}18- \\
25 \mathrm{yrs}\end{array}$ & 13.8 & 6.5 & 6.4 & 6.4 & 6.4 & 6.4 \\
\hline 29 & $\begin{array}{l}18- \\
25 \mathrm{yrs}\end{array}$ & 15.2 & 5.2 & 5.4 & 5.2 & 5.2 & 5.2 \\
\hline 30 & $\begin{array}{l}18- \\
25 \mathrm{yrs}\end{array}$ & 15.6 & 5.3 & 5.3 & 5.3 & 5.3 & 5.3 \\
\hline 31 & $\begin{array}{l}18- \\
25 \mathrm{yrs}\end{array}$ & 16.1 & 4.7 & 4.6 & 4.6 & 4.4 & 4.6 \\
\hline 32 & $\begin{array}{l}18- \\
25 \mathrm{yrs}\end{array}$ & 16.4 & 4.8 & 4.7 & 4.8 & 4.8 & 4.8 \\
\hline 33 & $\begin{array}{l}18- \\
25 \mathrm{yrs}\end{array}$ & 12.2 & 6.8 & 6.8 & 6.8 & 6.8 & 6.8 \\
\hline 34 & $\begin{array}{l}18- \\
25 \mathrm{yrs}\end{array}$ & 12.4 & 6.7 & 6.6 & 6.7 & 6.7 & 6.7 \\
\hline 35 & $\begin{array}{l}25- \\
30 \mathrm{yrs}\end{array}$ & 14.3 & 6.2 & 6.1 & 6.1 & 6.1 & 6.1 \\
\hline 36 & $\begin{array}{l}25- \\
30 \mathrm{yrs}\end{array}$ & 14.8 & 5.9 & 5.5 & 5.8 & 5.8 & 5.8 \\
\hline 37 & $\begin{array}{l}25- \\
30 \mathrm{yrs}\end{array}$ & 13.1 & 6.1 & 6.2 & 6.2 & 6.2 & 6.2 \\
\hline 38 & $\begin{array}{l}25- \\
30 \mathrm{yrs}\end{array}$ & 11.3 & 7.1 & 7.2 & 7.2 & 7.2 & 7.2 \\
\hline 39 & $\begin{array}{l}25- \\
30 \mathrm{yrs}\end{array}$ & 10.2 & 7.6 & 7.5 & 7.7 & 7.5 & 7.5 \\
\hline 40 & $\begin{array}{l}25- \\
30 \mathrm{yrs}\end{array}$ & 12.6 & 7.3 & 7.2 & 7.3 & 7.2 & 7.3 \\
\hline
\end{tabular}

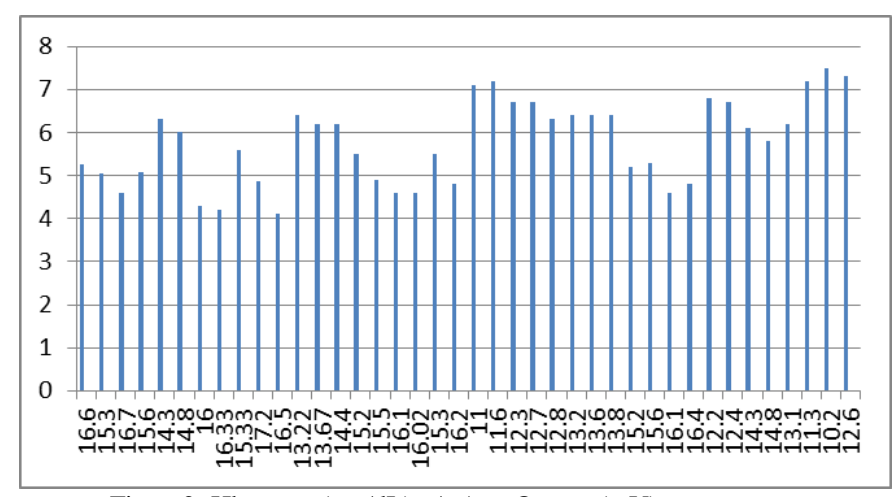

Figure2: Hb count (mg/dL) v/s Avg Output (mV)

\section{Entries based on Age group}

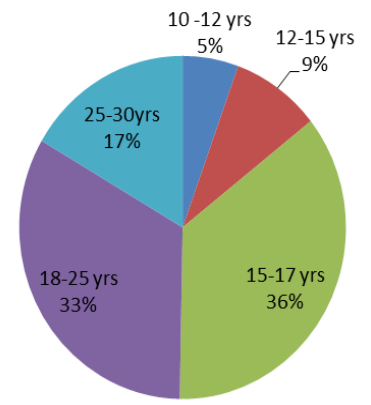

Figure3: Entries of different age group

\section{E. Result discussion}

- For women category having haemoglobin level between 12 -15.5 grams per decilitre is considered normal. Values below 12-13 grams per decilitre is considered to be iron deficit anaemia in females

- Similarly the hemoglobin level can also be used to detect the hemoglobin related diseases during pregnancy. The value below 12 grams per decilitre will be considered iron deficit and will have voltage range between $6.8 \mathrm{mV}-7.0 \mathrm{mV}$ and below 10 grams per decilitre is anaemic with voltage range between $7 \mathrm{mV}-8 \mathrm{mV}$.

- The haemoglobin level intensity can also help in finding the heart rate of a patient.

\section{CONCLUSION}

\section{A. General conclusion}

As the hemoglobin increases output voltage value reduces and thus inversely propotional. Higher the hemoglobin count higher will be the rate of the IR absorption,lower will be the output of photodetector and hence less output voltage. Interference of light with the samples could cause error in readings.

B. Future scope

By using fuzzy logic the device could be enhanced to describe the medications for anaemic and polycythemic subjects. 
Same principle could also be used for monitoring cholesterol and glucose level [3] and also to find the heart rate of a patient.

\section{REFERENCES}

[1] Sandeep Patil HG,Dr. Ram Kumar PS,Dr.G.K.Prabhu,Dr.AGIT N Babu, "Methods and devices to determine $\mathrm{Hb}$ non-invasively; a review, july 2014

[2] Rajashree Doshi, Anagha Pandit Rao,Optical sensor system for Hemoglobin Measurement.

[3] A.Kavithamani1 \& V. Manikandan2 Non invasive Hemoglobin leve and heart rate sensor system for Automatic treadmill speed control

[4] Aryeh Shander, Lawerence T.Goodnough, Mazyar Javidroozi, Michael Auerbach, Jeffrey Carson, William B. Ershler, Mary Ghiglione, John Glaspy and Indu Lew, - Iron Deficiency Anemia - Bridging the Knowledge and Practice Gapl, Journal of Transfusion Medicine
Reviews, Elsevier, vol.28, Issue 3, July 2014, pp.156-166, doi:10.1016/j.tmrv.2014.05.001.

[5] Lipinski MJ, Dewey FE, Biondi-Zoccai GG, Abbate A, Vetrovec GW and Froelicher VF, - Hemoglobin levels predict exercise performance, STsegment depression, and outcome in patients referred for routine exercise treadmill testing\|, Journal of Clinical Cardiology, vol. 32, Issue 12, pages E22 - E31, December 2009.

[6] Jens Kraitl, Ulrich Timm, Hartmut Ewald and Elfed Lewis, -Noninvasive measurement of blood componentsl, Proceedings of Fifth International Conference on Sensing Technology. pp.253-257, 2011.

[7] Rajashree Doshi and Anagha Panditrao, - Noninvasive optical sensor for Hemoglobin Determinationl, International Journal of Engineering Research and Applications (IJERA) vol. 3, Issue 2, pp.559-562, March -April 2013, v ISSN: 2248-9622. 